\title{
Thoracic Irradiation Recruit M2 Macrophage into the Lung, Leading to Pneumonitis and Pulmonary Fibrosis
}

\author{
Hae-Ran Park*, Sung-Kee Jo, Uhee Jung \\ Radiation Division for Biotechnology, Advanced Radiation Technology Institute, Korea Atomic Energy Research Institute, Jeongeup, Korea
}

\section{Original Research}

Received August 22, 2017

Revision October 30, 2017

Accepted November 3, 2017

Corresponding author: Hae-Ran Park

Radiation Division for Biotechnology, Advanced Radiation Technology Institute, Korea Atomic Energy Research Institute, 29 Geumgu-gil, Jeongeup 56212, Korea Tel: +82-63-570-3222

Fax: +82-63-570-3229

E-mail: hrpark@kaeri.re.kr

This is an Open-Access article distributed under the terms of the Creative Commons Attribution NonCommercial License (http://creativecommons.org/ licenses/by-nc/4.0) which permits unrestricted noncommercial use, distribution, and reproduction in any medium, provided the original work is properly cited.

Copyright $\odot$ 2017The Korean Association for Radiation Protection

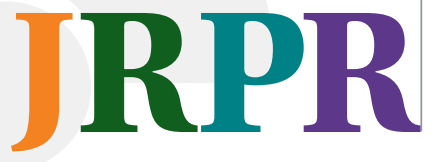

Background: Radiation-induced pneumonitis and pulmonary fibrosis are common dose-limiting complications in patients receiving radiotherapy for lung, breast, and lymphoid cancers. In this study, we investigated the characteristics of effective immune cells related to pneumonitis and fibrosis after irradiation.

Materials and Methods: After anesthesia, the whole thorax of C57BL/6 mice was irradiated at 14 Gy. The lung tissue and bronchoalveolar lavage fluid were collected at defined time points post-irradiation for the determination of histological and immunohistochemical analysis and inflammatory cell population infiltrated into the lung.

Results and Discussion: Whole thoracic irradiation increased the deposition of extracellular matrix (ECM), lung weight, and pleural effusions, which started to die from 4 months later. At 4 months after irradiation, the numbers of macrophages and lymphocytes as well as neutrophils were increased dramatically in the lung. Interestingly, the macrophages that were recruited into the lung after irradiation had an enlarged foamy morphology. In addition, the expressions of chemokines (CCL-2, CCL-3, CXCL-10) for the attraction of macrophages and T cells were higher in the lung of irradiated mice. The high expressions of these chemokines were sustained up to 6 months following irradiation. In thoracic irradiated mice, infiltrated macrophages into the lung had the high levels of Mac-3 antigens on their surface and upregulated the hallmarks of alternatively activated macrophages such as arginase- 1 and CD206. Furthermore, the levels of IL-4 and IL-13 were higher in a BAL fluid of irradiated mice.

Conclusion: All results show that thoracic irradiation induces to infiltrate various inflammation-related immune cells, especially alternatively activated macrophages, through enhancing the expression of chemokines, suggesting that alternatively activated macrophages are most likely important for leading to pulmonary fibrosis.

Keywords: Thoracic irradiation, Pneumonitis, Fibrosis, Inflammatory immune cells, Alternatively activated macrophages

\section{Introduction}

Thoracic radiation therapy is used to treat lung, breast, and lymphoid cancers. However, radiation-induced pneumonitis and pulmonary fibrosis are common dose-limiting comp0lications in radiotherapy [1]. Although there are cytokine-mediated multicellular interactions with induction and progression of fibrotic tissue reactions as indicated based on many researches concerning the incidence mechanisms of pulmonary fibrosis after irradiation, the mechanisms underlying the pathogenesis of radiation-in- 
duced pulmonary fibrosis at the molecular and cellular levels have not yet been identified.

Fibrosis is defined by the excessive accumulation of extracellular matrix (ECM) components such as a mixture of proteins (collagen [especially type I and III] and elastin), glycoproteins and proteoglycans (fibronectin, laminin, and tenascin) in and around inflamed or damaged tissue through an imbalance of ECM synthesis and degradation [2, 3]. ECM is produced by activated myofibroblasts, which are differentiated from fibroblast, resident mesenchymal cells, epithelial and endothelial cells after exposure to the fibrogenic cytokines released by inflammatory immune cells. Because ECM-producing myofibroblasts are central to fibrosis, fibrosis research has focused on explaining the mechanisms that initiate, maintain and terminate the differentiation of fibroblasts into actively proliferating, ECM-producing myofibroblasts [2-4]. However, we have concentrated on the effective immune cells that participate in lung inflammation and fibrosis after thoracic irradiation, because the differentiation and activation of fibroblasts are regulated by many elements of the innate and adaptive immune response $[5,6]$.

Generally, chronic inflammation is typically characterized by triggering the recruitment of various immune cells into the lung, including macrophages, lymphocytes, eosinophils and neutrophils. It is very important for the wound-healing process that neutrophils and monocytes/macrophages are recruited quickly after damage and participate in the removal of debris from damaged tissue. However, because these cells also secrete a variety of toxic mediators that are harmful to the surrounding tissues, if macrophages and neutrophils that were infiltrated into the inflammatory site are not quickly eliminated, they can further exacerbate the tissue-damaging inflammatory response that leads to fibrosis. Among the various inflammatory cell types, macrophages are the main effecter cells involved in the pathogenesis of fibrosis as well as critical in regulating host response to lung injury because they are the first line of defense against pathogens. Duffield and his colleagues reported that macrophage depletion at an early stage of inflammation was shown to substantially reduce the development of liver fibrosis in mice [7].

Chemokines are leukocyte chemoattractants that play an important role in the recruitment of inflammatory cells to damaged sites. The chemokines are classified on the basis of the number and location of $\mathrm{N}$-terminal cysteine residues: the CC chemokines, in which the cysteine residues are adjacent, and the CXC chemokines, in which these residues are separated by one amino acid. To control the nature of the inflammatory infiltrate, different chemokines act on different cells. Representative chemokines for macrophage recruitment are CCL2 and CCL3, while CXCL-10 acts mainly on T cells and macrophages. Johnston and colleagues published that various chemotactic activities for neutrophils, macrophages, and lymphocytes occur during pneumonitis after irradiation $[8,9]$.

Recently, we reported that whole body fractionated irradiation leads to chronic allergic airway inflammation by increasing the influx of macrophages in allergen-injected mice [10]. There are also previous studies concerning the relationship between macrophages and pulmonary fibrosis in thoracic irradiated murine models [11-15]. Peripheral blood monocytes/macrophages migrate to the inflammation site and result in activation and polarization according to the micro-environment in the lung. Macrophage subsets with distinct functions have been described [16, 17]: classically activated macrophages (CAMs; M1) that are mainly activated by Toll-like receptor ligands and Thl cytokines such as IFNgamma, and alternatively activated macrophages (AAMs; M2) that are stimulated by Th2 cytokines such as IL-4 and IL-13 and characterized by an elevated expression of arginase type 1 (Arg-1), macrophage mannose receptor (MMR, CD206), resistin-like molecule- $\alpha$ (FIZZ-1), CCL18, and CXCL13 [18-20]. The classical mechanisms of activating macrophages have been proposed to play an important role in generating pro-inflammatory cytokines and inducing radiation pneumonitis $[3,6]$. Whereas alternatively activated macrophages are responsible for the secretion of anti-inflammatory cytokines and indispensable for tissue remodeling and often associated with fibrosis [3, 6, 14]. Interestingly, the alternatively activated macrophages express the characteristic marker proteins involved in profibrotic changes, such as up-regulated L-arginine metabolism by Arg- 1 to generate polyamines and proline, the generation of growth factors, including TGF- $\beta 1$ and PDGF, that stimulate fibroblasts and epithelial cells, and alteration in ECM.

Thoracic irradiation affects not only the macrophages but also various immune cells in the lung tissue. Many studies are still necessary to define how the various immune cells interplay and are coordinated during radiation-induced fibrosis. In the current study, we evaluated that thoracic radiation induced the recruitment of various immune cells, especially enlarged foamy macrophages, into the lung via enhancing the expression of chemokines. Moreover, we examined the 
phenotypic characteristics of macrophages that were infiltrated into the lung after thoracic irradiation.

\section{Materials and Methods}

\section{Animals and Ethics Statement}

Six-week-old female C57BL/6N (H-2 $\left.{ }^{\text {b }}\right)$ mice were purchased from Orient Inc. (Charles River Technology; Seoul, Korea). The mice were housed at a controlled temperature of $22 \pm 2^{\circ} \mathrm{C}$ and at $50 \pm 5 \%$ relative humidity and under a specific pathogen-free condition. The mice were fed with a standard animal diet and water ad libitum. The animal experiments were conducted according to the principles enunciated in the 'Animal Care Act', prepared by the Ministry of Agriculture and Forestry, Republic of Korea. The protocol was approved by the Korea Atomic Energy Research Institute Institutional Animal Care and Use Committee (KAERI IACUC) and included criteria for euthanasia to minimize suffering. The number of mice used for each analysis is shown in the figure legends.

\section{Thoracic irradiation}

The mice were irradiated once with 14 Gy using a Gammacell 40 lead collimator with a ${ }^{137} \mathrm{Cs} \gamma$-ray source (Gammacell 40 Exactor, Nordin International Inc. Ottawa, Canada) at a $0.6 \mathrm{~Gy} / \mathrm{min}$ dose rate. The mice were anesthetized with Zoletil (20 mg/kg body weight, Virbac S.A, France) just before irradiation and then were confined in specifically designed plastic jigs and placed such that the thoraces were in a $3 \mathrm{~cm}$ diameter field defined by lead collimators. Age-matched normal controls were maintained under identical conditions for the course of the experiment.

\section{Survivability and lung weight after irradiation}

After thoracic irradiation, the survivability was checked every day during 24 weeks. Survival was calculated as the percentage of mice that did not require euthanasia. We observed more carefully on and after 16 weeks following thoracic irradiation, because mice started to die.

\section{Determination of the volume of pleural exudates}

The chest was carefully opened, and the plural cavity was washed with $0.5 \mathrm{~mL}$ of PBS with heparin $(5 \mathrm{U} / \mathrm{mL})$. The exudates and washing solution were gently aspirated using a 3 $\mathrm{mL}$ syringe, and the total volume was measured. The results were calculated by subtracting the volume injected $(0.5 \mathrm{~mL})$ from the total volume recovered.

\section{Masson's trichrome blue staining}

The lungs were fixed in $10 \%$ neutral-buffered formalin, dissected, embedded in paraffin, and cut into $5 \mu \mathrm{m}$ thick sections. The sections were stained with Masson's trichrome blue (Diagnostic BioSystems; Pleasanton, CA, USA) to determine the deposition of peribronchial and interstitial collagen according to the manufacturer's directions. Briefly, deparaffinized slides were staining with bouins fluid, weighrt's hematoxylin, biebrich scarlet-acid fuchsin, phosphomolybdic/ phosphotungstic acid, and then aniline blue stain in series. Finally, these slides were dehydrated and substituted into Xylene. The slides were mounted and observed by light microscopy $(\times 400)$.

\section{6. cDNA microarray analysis}

The total RNA was isolated from the lung tissue using the homogenizer in a TRIzol Reagent (Invitrogen, Carlsbad, California, USA). The cDNA then underwent second-strand synthesis and clean-up to become a template for in vitro transcription with T7 RNA polymerase and biotin UTP, which generates multiple copies of biotinylated cRNA was carried out for 14 hours. After purification, the purity and concentration of cRNA was cheked using a BioRad's Experion Automated Electronphoresis System (Bio-Rad Laboratories, Inc.; Seoul, Korea). High quality cRNA was then used with Illumina direct hybridization array kits. cRNA $(0.75 \mu \mathrm{g})$ was hybridized on Mouse WG-6 v2.0 Expression BeadChip (Illumina, San Diago, CA, USA) at $58^{\circ} \mathrm{C}$ for 16 hours in a multiple step procedure according to the manufacturer's instructions. Hybridization was detected with $1 \mathrm{mg} / \mathrm{mL}$ Cy3-streptavidin (GE Healthcare). The chips were then washed, dried, and scanned on the Bead Array Reader (Illumina), and raw data were generated using GenomeStudio, and then normalized using the Quantile algorithm. Gene expression profiles were statistically compared using the "Differential Expression" feature of GenomeStudio with $t$ test.

\section{Preparation of total leukocytes from bronchoalvelolar} lavage (BAL) fluid and differential counting

Mice were sacrificed by cervical dislocation at 2 and 4 months after thoracic irradiation, respectively. The trachea was cannulated and BAL was obtained by washing the airway lumina. Briefly, total cells in the lungs were recovered by flushing three times with $0.5 \mathrm{~mL}$ of PBS including with $1 \mathrm{mM}$ 
EDTA and 10\% FBS through a tracheal cannula. The total leukocytes were recovered in PBS including 10\% FBS after removing the RBC using a RBC lysis buffer (Sigma-Aldrich Co., St Louis, MO, USA) and then were smeared using cytospin centrifuge (Hanil; Gangwondo, Republic of Korea; $400 \mathrm{~g}$ for 4 minutes). Differential cell counts were performed after staining with a Diff-Quick stain Set (Sysmex; Kobe, Japan). A minimum of 500 cells per slide were counted, and the cells were classified as macrophages, lymphocytes, neutrophils, or eosinophils by morphological criteria. The supernatant of BAL fluid was stored at $-30^{\circ} \mathrm{C}$ for determination of cytokine levels.

\section{Preparation of total leukocytes from the lung tissue and flow cytometry analysis}

The mice were killed at 2, 4, and 6 months after thoracic irradiation, respectively. The lungs were minced in a digestion medium containing $100 \mathrm{U} / \mathrm{mL}$ collagenase (Gibco). After incubation for 30 minutes at $37^{\circ} \mathrm{C}$, the digestion solution was passed through a $70 \mu \mathrm{m}$ mesh strainer and the total leukocytes were recovered in PBS after removing the RBC using an RBC lysis buffer (Sigma).

Immunophenotyping of the total leukocytes from the lung tissues was carried using a FACSCaliburTM flow cytometer (Beckman Coulter; Miami, Florida, USA). An analysis was conducted by the selection of total leukocytes on the basis of the forward and side scatter profile. A fluorescence histogram of at least 10,000 counts was counted in each sample. The monoclonal antibodies (BD Pharmingen, SanDiego, CA, USA), labeled with either fluorescein isothiocyanate (FITC) or phycoerythrin (PE), were directed against the following: $\mathrm{CD}$ (cluster of differentiation) 19, B cells; CD3, total T cells; CD11b, total macrophages; Mac-3, activated macrophages. Cells were resuspended in a staining buffer (4\% FBS and $0.1 \%$ sodium azide in PBS) and Fc receptors blocked with anti-CD16/CD32 monoclonal antibody (Mouse BD Fc Block ${ }^{\mathrm{TM}}$, BD Pharmingen, SanDiego, CA, USA) for 20 minutes at $4^{\circ} \mathrm{C}$. To obtain the absolute number of cells for each lymphocyte population, the following formula was used: no. of cells in the lung $=$ no. of total leukocytes/lung $\times$ percentages of population.

\section{Immunohistochemistry analysis}

The lungs were fixed in $10 \%$ neutral-buffered formalin, dissected, embedded in paraffin, and cut into sections $5 \mu \mathrm{m}$ in thickness. To detect the activated macrophages in the lung, the sections were stained with anti-mouse Mac-3 anti- body (BD Pharmingen) using a streptavidin (SAv)/biotin staining method system (BD Pharmingen) according to the manufacturer's directions (www.bdbiosciences.com). Antimouse CD16/CD32 monoclonal antibody was used for the blocking of Fc-mediated binding of antibodies (Mouse BD Fc Block ${ }^{\mathrm{TM}}$, BD Pharmingen, SanDiego, CA, USA). Finally, the slides were incubated with a DAB substrate, counterstained with haematoxylin, dehydrated, mounted and studied by light microscopy $(400 \times)$. All incubations were carried out under humidified conditions.

\section{Analysis of the mRNA expression in the reverse transcriptase (RT)-polymerase chain reaction (PCR)}

To extract the total RNA from the right half of the lung, the lung was homogenized with TRIzol Reagent (Invitrogen, California, USA). Reverse transcription was implemented, and cDNA was synthesized from $3 \mu \mathrm{g}$ of total RNA using random primer and MMLV reverse transcriptase (Promega, Madison, WI, USA). cDNAs of interest were amplified from a $1 \mu \mathrm{L}$ RT product per PCR using the Taq DNA polymerase (Promega, Madison, WI, USA). The primers were as follows: $\beta$-actin, $5^{\prime}$ AGCCATGTACGTAGCCATCC-3' and 5'-TCTCAGCTGTGGTGGTGAAG-3'; CCL-2, 5'-CCCAATGAGTAGGCTGGAGA-3' and 5'-AGACCTTAGGGCAGATGCAG-3'; CCL-3, 5' -ACCATGACCTCTGCAACCA-3' and 5'-CCCAGGTCTCTTTGGAGTCA-3'; CXCL-10, 5'-AAGTGCTGCCGTCATTTTCT-3' and 5'-TTCATCGTGGCAATGATCTC-3'; CD32, 5'-TGTCGCAGCCATTGTTATTATC- ${ }^{\prime}$ ' and $5^{\prime}$-TGTGGTTCTGGTAATCATGCTC-3'; Arg-1, 5'-CAGAAGAATGGAAGAGTCAG-3' and 5'CAGATATGCAGGGAGTCACC-3'; CD206, 5' -ACGACAATCCTGTCTCCTTTGT-3' and 5'-TCAGCTTTGGTTGTAATGGATG-3'. Amplification of the $\beta$-actin (22 cycles), CCL-2 (31 cycles), CCL-3 (32 cycles), CXCL-10 (31 cycles), CD32 (32 cycels), Arg-1 (31 cycles), and CD206 (29 cycles) were performed for 20 s at $95^{\circ} \mathrm{C}, 30$ s at $60-64^{\circ} \mathrm{C}$, and 40 s at $72^{\circ} \mathrm{C}$ in a thermal cycler (Applied Biosystems; California, USA). PCR products were resolved on $1.5 \%$ agarose gel by electophoresis and were quantitated by a Gel Imaging system (Gel DocTM ${ }^{\mathrm{XR}}+$ Imager; Bio-Rad Laboratories, Inc.).

\section{Western blotting}

Total proteins from the lung tissue were obtained by homogenizing in a cold tissue lysis buffer containing $10 \mathrm{mM}$ Tris-HCl, pH 7.0, 100 mM NaCl, 5 mM EDTA (ethylenediaminetetraacetic acid), $10 \%$ Glycerol, $1 \%$ Nonidet P-40, and a 
protease inhibitor cocktail (Sigma-Aldrich Co.). The protein concentrations of the cell extracts were measured using the BCA (bicinchoninic acid) method (Pierce, Rockford, USA). Protein $(30 \mu \mathrm{g})$ was boiled in a Laemmli sample buffer containing mercaptoethanol (Bio-Rad Laboratories, Inc.) and then separated on SDS-polyacrylamide gel and then transferred to a Hybond ${ }^{\mathrm{TM}}$-P membrane (Amersham, Seoul, Korea). After blocking in a blocking buffer ( $50 \mathrm{~g} / \mathrm{L}$ skimmed milk in TBST [tris-buffered saline tween-20] containing $1 \mathrm{~mL} /$ L tween-20), the membranes were incubated with primary antibodies, anti-iNOS antibody (abcam; Gyeonggi-do, Korea), anti-Arg- 1 antibody (abcam) and anti- $\beta$-actin antibody (Cell signaling; Banvers, MA, USA), and then with anti-Goat IgG antibody conjugated with horseradish peroxidase (HRP) or anti-rabbit IgG antibody conjugated with HRP (Cell Signaling). Chemiluminescence was detected according to the manufacturer's protocol (Millipore, Billerica, MA, USA).

\section{Measurement of cytokines in BAL fluid}

The levels of IL-4 and IL-13 in BAL fluid were measured by ELISA according to the manufacturer's instructions (www. bdbiosciences.com or www.RnDSystems.com/MAPELISA). Antibodies as follows were used: For the IL-4, clone BVD4-
1D11 was the capture antibody, and biotin-labelled BVD624G2 was the detecting antibody. For the IL-13, clone 38213 and biotin-labelled anti-mouse IL-13 antibody was used as the capture and the detecting antibody, respectively. All these antibodies for IL-4 detection and the recombinant IL-4 were purchased from BD PharMingen (SanDiego, CA) and antibodies for IL-13 as well as the recombinant IL-13 were purchased from R\&D Systems (Minneapolis, MN).

\section{Statistical analysis}

Data were expressed as mean \pm S.D. and a statistical significance of the differences between two groups (control mice vs. thoracic irradiated mice) was analyzed by using a Student's $t$ test with a two-tail test. A $p$-value of $<0.01$, or $<0.05$ was considered as statistically significant.

\section{Results and Discussion}

\section{Thoracic irradiation-induced pneumonitis and pulmonary fibrosis}

Jackson and colleagues presented an actual report on radiation-induced pneumonitis and fibrosis in mice strains: The C57L mice were sensitive to early pneumonitis at 3 to 4
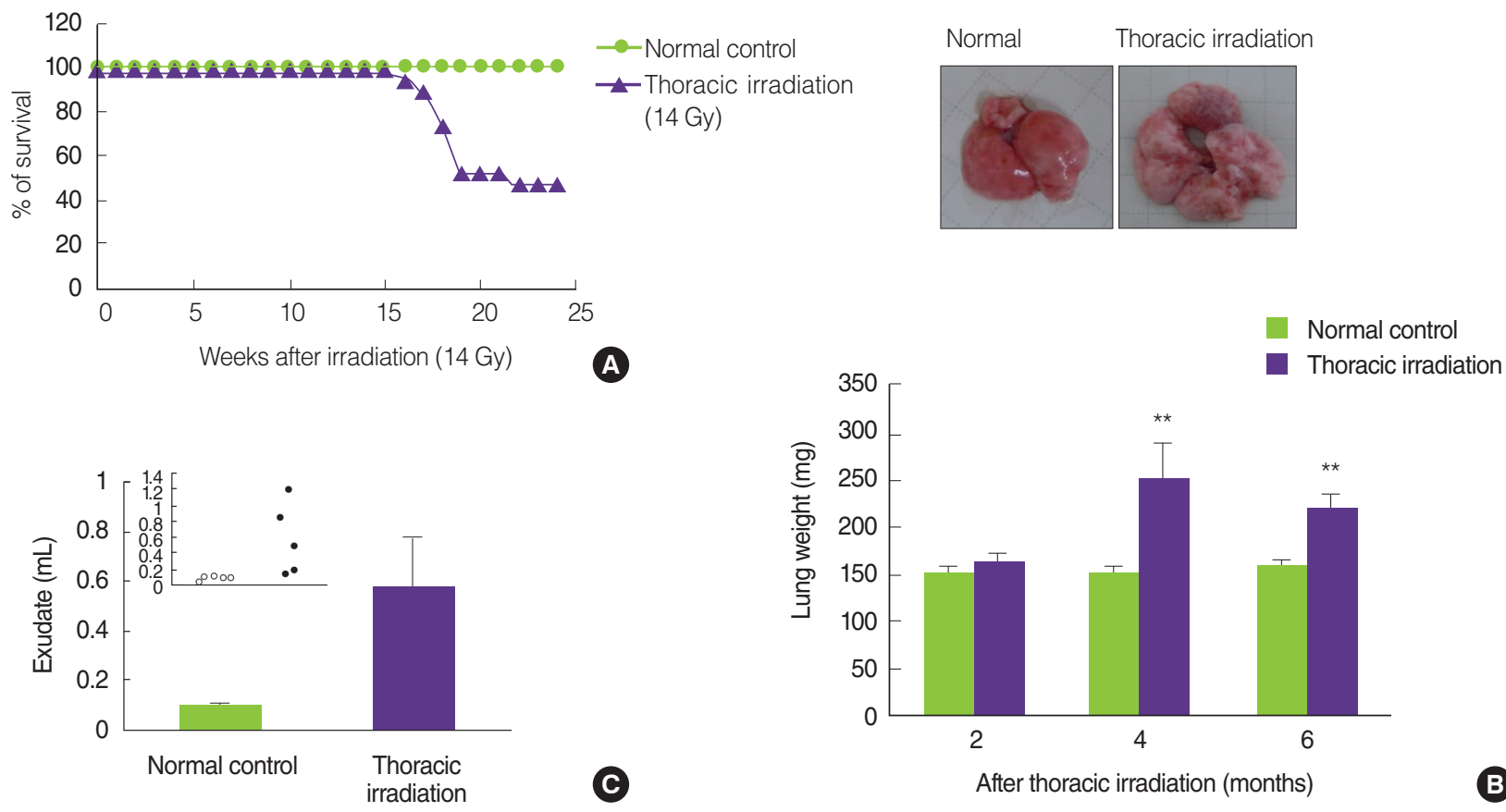

Fig. 1. Thoracic irradiation induces pneumonitis and pulmonary fibrosis. (A) The survivability was checked during 6 months after thoracic irradiation of 14 Gy. There were nineteen mice in each group. (B) At 2, 4, and 6 months later, mice were sacrificed, and then lung tissues were weighed. There were five mice in each group. The pictures show the lung tissue at 6 months after irradiation. Data shows the mean \pm S.D. (C) At 6 months later, pleural exudates were gently aspirated using a syringe and its volume was measured. There were five mice in each group. Data shows the mean \pm S.E. ${ }^{*} p<0.05 ;{ }^{* *} p<0.005$ compared to the age-matched control mice. 
months while C57BL/6 mice showed a delayed response, with most mice presenting with large accumulations of pleural fluid at 6 to 9 months [21]. Strain-dependent variation is shown in pneumonitis and fibrotic response through a difference in the levels of TGF- $\beta$, TNF- $\alpha$, IL- $1 \alpha / \beta$, and IFN- $\gamma$; Namely, C3H mice develop only classical pneumonitis during the early phase, whereas C57BL/6 mice undergo fibrosis during the latent period [6, 22-24]. Mice strain was C57BL/6N and the survivability after thoracic irradiation of 14 Gy was checked every day during 6 months. As shown in Figure 1A, thoracic irradiated mice started to die at 4 months and the survival rates were $47.3 \%$ at 6 months after irradiation. We measured the increased weight of the lung and pleural exudates as the signs of the pneumonitis and pulmonary fibrosis. Compared with the age-matched normal control, the lung weights of the thoracic irradiated mice were significantly increased at 4 months and 6 months, but not at 2 months (Figure $1 B$ ). In addition, the volumes of pleural fluid were significantly increased at 6 months following thoracic irradiation (Figure 1C). These results suggested that thoracic irradiated mice are killed because of pneumonitis and lung fibrosis.

\section{Thoracic irradiation induces the accumulation of extracellular matrix in the lung}

To determine whether the thoracic irradiation affected the matrix regulation, paraffin-embedded sections of the lung were stained with Masson's trichrome blue to show the collagen deposition at 2, 4, and 6 months after thoracic irradiation (Figure 2A; blue staining represents collagen). At 2 months later, collagen deposition around the airways was no difference between normal control groups and thoracic irradiation groups. However, the extent of collagen deposition around the airways of and in interstice of the lung of thoracic irradiated mice was more extensive at 4 and 6 months later. In particular, mice at 6 months after irradiation remarkably increased the collagen deposition in interstice of the lung. Next, we confirmed that thoracic irradiation induced to increase the mRNA expression levels of type I collagen in the lung through a cDNA microarray analysis. As can be seen in Figure $2 \mathrm{~B}$, the expression levels of type I collagen in the lung of thoracic irradiated mice were markedly increased at 4 months and reached the highest levels at 6 months after thoracic irradiation. The expression of Coll $\alpha 2$ was not different statistically at 2 months after thoracic irradiation.

\section{Thoracic irradiation increases the recruitment of various immune cells into BAL fluid and the lung}

Many reports have indicated that thoracic irradiation dominantly induces the recruitment of macrophages and lymphocytes during pneumonic and fibrotic phages [12, 25, 26]. At histological analysis as shown in Figure 2A, we observed infiltration of leukocytes into the lung after irradia-

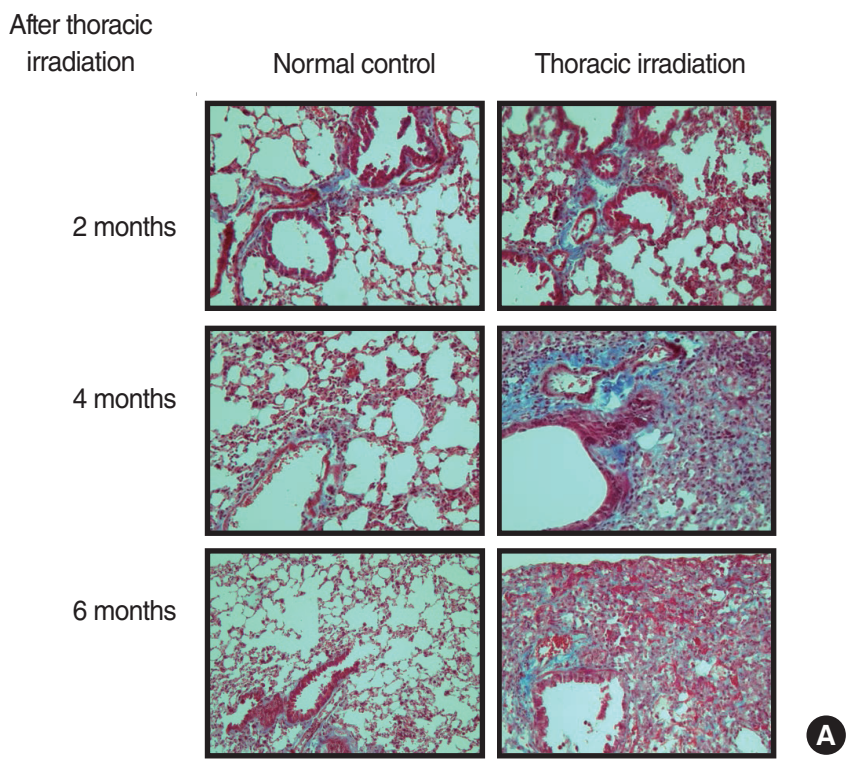

Col $1 \alpha 2$

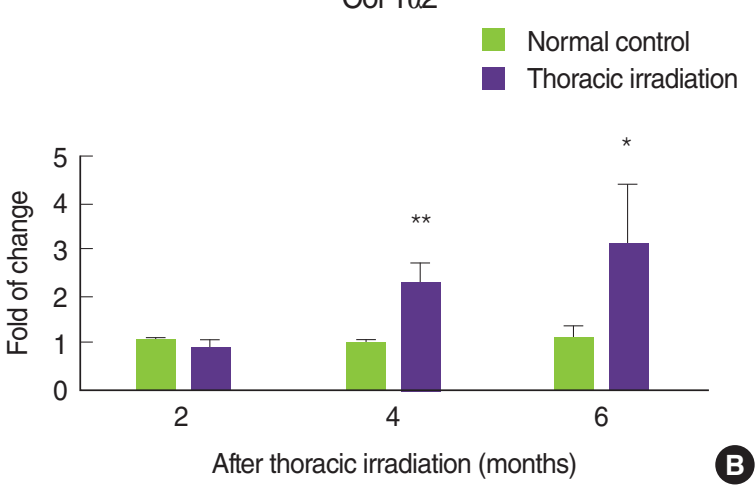

Fig. 2. Effect of thoracic irradiation on the collagen deposition in the lung. At 2, 4, and 6 months after thoracic irradiation, collagen deposition (A) and the mRNA expression patterns of collagen type 1a2 (B) in the lung was determined by Masson's trichrome blue staining and microarray analysis. ${ }^{*} p<0.05 ;{ }^{* *} p<0.01$ compared to the age-matched control mice. 
tion, especially at 4 and 6 months later (cytoplasm was stained by red). Therefore, we counted the cells that were recruited into the BAL fluid at 2 and 4 months after irradiation. Compared with the normal control, many numbers of total cells were observed in the BAL fluid of thoracic irradiated mice, and the potential for gathering the cells was more enhanced at 4 months after irradiation than at 2 months (Figure $3 \mathrm{~A})$. Next, we differentially counted the macrophage, lymphocyte, eosinophil and neutrophil of the total cells in BAL fluid (Figure 3B). At 2 months after irradiation, the number of neutrophils was increased significantly in thoracic irradiated mice when compared with that of normal control mice (Figure 3C). Eosinophils in the BAL fluid of all groups were negligibly observed. The recruitment of macrophages and lymphocytes were increased at 4 months after thoracic irradiation. Interestingly, the morphology of macrophages in thoracic irradiated mice was changed into enlarged foamy macrophages when compared with that of normal control mice as shown in Figure 3B, and macrophages in thoracic ir- radiated mice were then markedly increased (Figure 3C). Next, we checked the type of lymphocytes that were infiltrated into the lung using flow cytometric analysis. The numbers of B cells (CD19+CD3-) were significantly increased at 4 and 6 months after irradiation and the T cells (CD3+CD19-) and total macrophages $(\mathrm{CD} 11 \mathrm{~b}+)$ were significantly increased at 2 and 4 months (Figure 4 ) when compared to those of agematched normal mice.

\section{Thoracic irradiation enhances the chemokines expression for the attraction of macrophages}

Macrophages are one of the target cells in the pulmonary fibrosis because of the major producers of TGF- $\beta$, which are, indisputably, one of the key drivers of fibrosis. In addition, macrophages play an important role in fibroblast migration and differentiation/proliferation into myofibroblasts, which are the key mediators of fibrotic tissue remodeling [3, 5, 27]. Therefore, we concentrated on the recruitment of macrophages into the lung of thoracic irradiated mice. We exam-

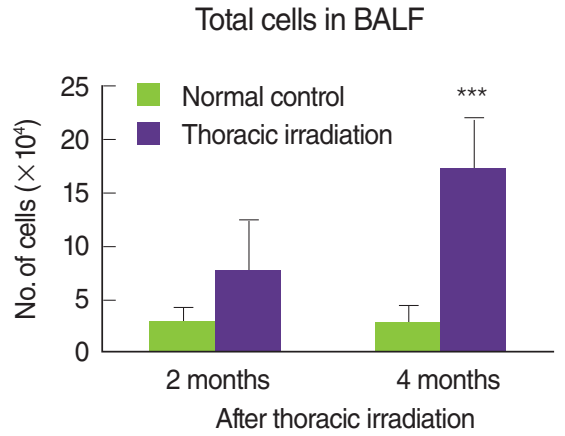

(A)

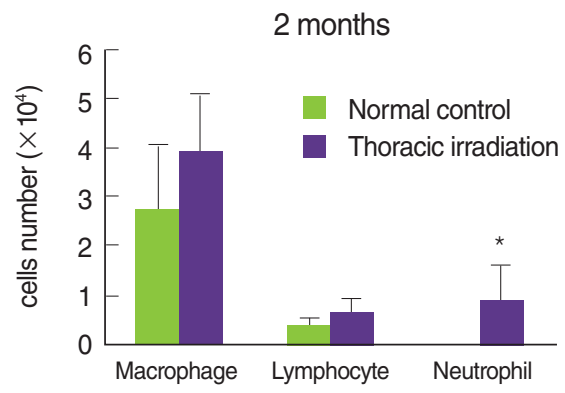

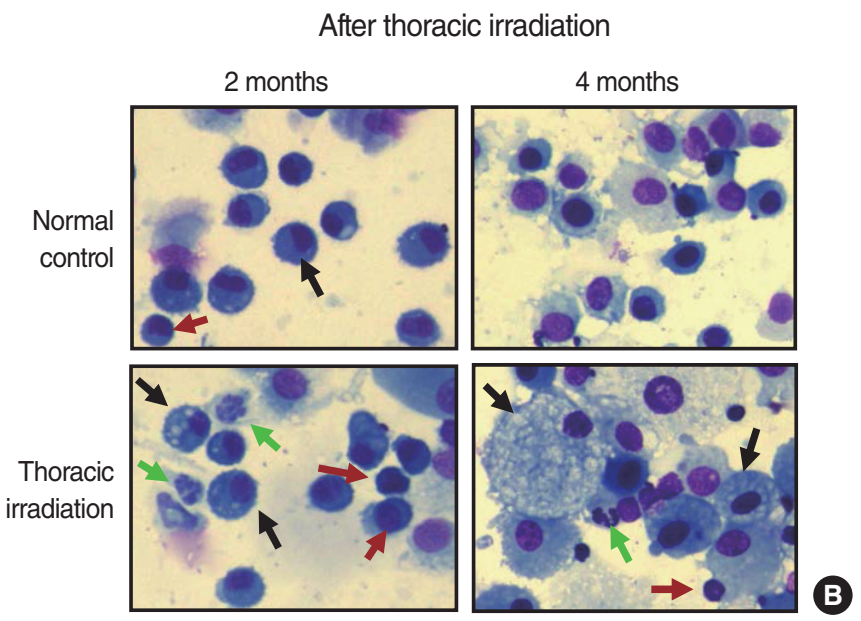

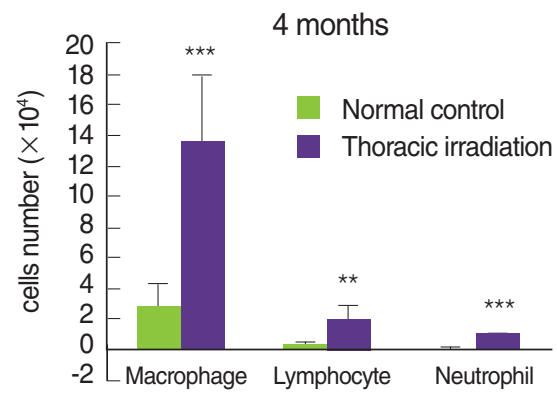

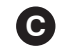

Fig. 3. Effect of thoracic irradiation on the recruitment of immune cells into the lung. At 2 and 4 months following thoracic irradiation, total cells in the BAL fluid were counted $(A)$ and then were smeared on slides. After staining with Diff-Quick stain, representative slides were showed (B). Black arrows, macrophages; red arrows, lymphocytes; green arrows, neutrophil. (C) At least 500 cells per slide were counted by the morphological criteria. There were six mice in each group. The bars indicate the means \pm SD. ${ }^{*} p<0.1 ;{ }^{* \star} p<0.005 ;{ }^{* \star *} p<0.001 \mathrm{compared}$ to the age-matched control mice. 
ined the expression levels of chemokine such as CCL2, CCL3, and CXCL10, which need to attract monocytes and macrophages. As shown in Figure 5, thoracic irradiation induced to significantly increase the mRNA expression of CXCL10, CCL2, and CCL3 in the lung at 2 months, and then high expression of these chemokines were sustained until 6 months when compared with that of age-matched normal control mice. At 1 month following thoracic irradiation, the expression of these chemokines was negligibly increased, but high expression of CXCL10 was only significant.
B cell $\left(C D 19^{+}\right)$

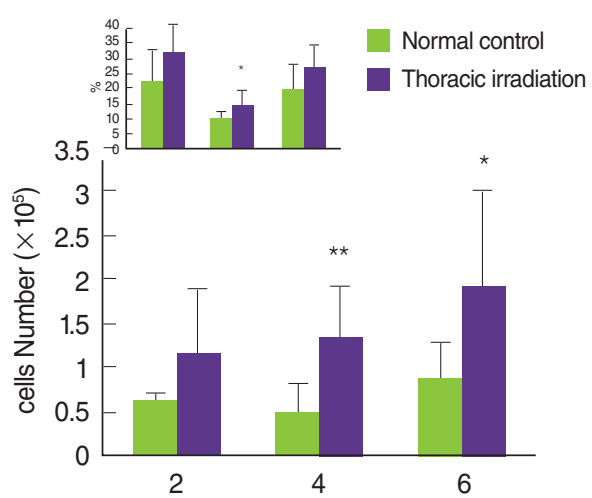

After thoracic irradiation (months)
Total T cell $\left(\mathrm{CD}^{+}\right)$

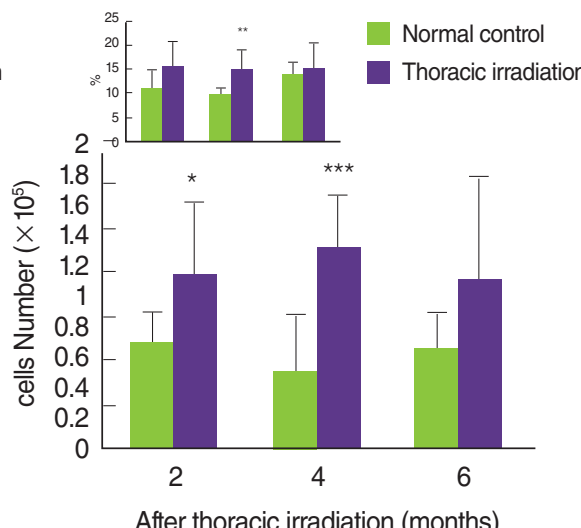

After thoracic irradiation (months)
Macrophage (CD11b+)

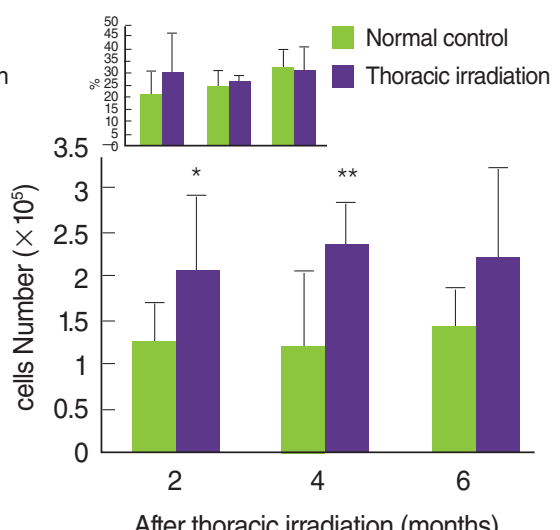

Fig. 4. Comparison of the number of B cells, $T$ cells and macrophages in the lung between thoracic irradiated mice and age-matched control mice. At 2, 4, and 6 months after thoracic irradiation, total leukocytes from the lung were preparation and then were analyzed by flow cytometer following staining with each mAb. To obtain the absolute number of cells for each cell population, the following formula was used: no. of cells in population/the lung $=$ no. of total leukocytes/the lung $\times$ percentages of population (insert). There were six mice in each group. The bars indicate the means $\pm \mathrm{SD}$. ${ }^{\star} p<0.1 ;{ }^{* \star} p<0.05 ;{ }^{* \star *} p<0.01$ compared to the age-matched control mice.

After thoracic irradiation

1 month

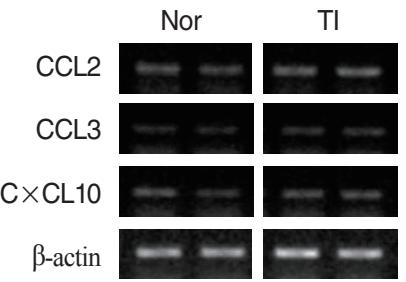

CCL2

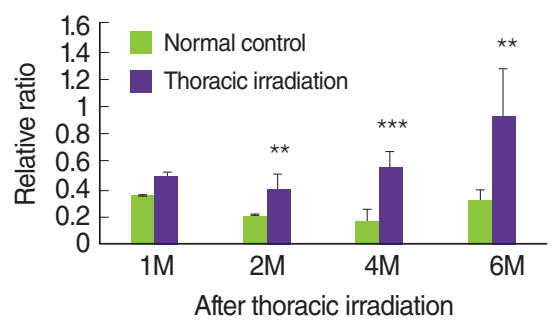

2 month

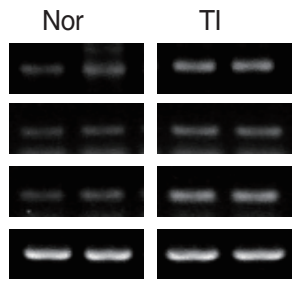

CCL3

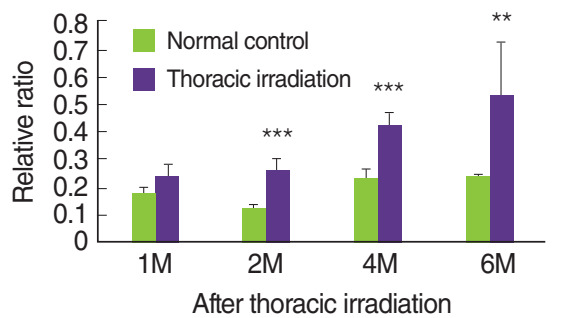

6 month
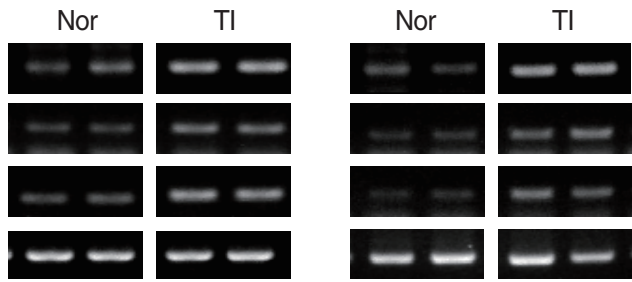

$\mathrm{C} \times \mathrm{CL} 10$

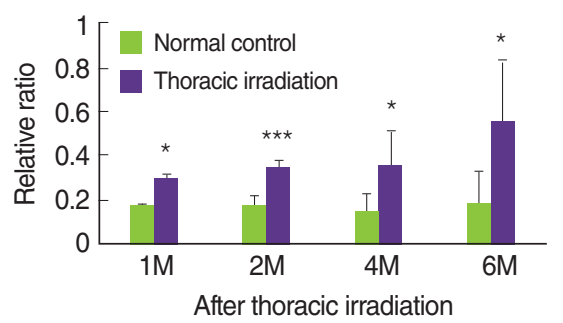

Fig. 5. Effect of thoracic irradiation on the mRNA expression of chemokines for the attraction of macrophages. At 1, 2, 4, and 6 months after thoracic irradiation, the mRNA expression of CCL2, CCL3, and CXCL10 in the lung was determined by RT-PCR methods and were quantitated by a Digital Gel Image system. The quantification was normalized to the reference gene $\beta$-actin. There were six mice in each group. The bars indicate the means \pm SD. ${ }^{*} p<0.05 ;{ }^{* *} p<0.01 ;{ }^{* *} p<0.001$ compared to the age-matched control mice. 


\section{Recruited macrophages after thoracic irradiation have Mac-3 antigen on their surface}

Infiltrating macrophages expressed CD11b (Mac-1) more highly than mature tissue macrophages [28]. More interestingly, Mac-3 antigens on their surface is commonly upregulated during macrophage activation and differentiation [29, 30]. Therefore, we determined whether or not thoracic irradiation induced the expression of Mac-3 on macrophages that are gathered into the lung after thoracic irradiation. After thoracic irradiation, $\mathrm{CD} 11 \mathrm{~b}+$ macrophages that were recruited into the lung showed to be decreased the ratio of Mac-3 negative (Figure 6A). In contrast, the ratio of macrophages that had Mac-3 antigen on their surface was increased after thoracic irradiation (Figure 6B). This phenomenon vividly appeared at 4 and 6 months after thoracic irradiation. In a further histological examination, we obtained a similar re- sult; namely, more Mac-3 positive macrophages were detected in the lung of thoracic irradiation groups when compared with the normal control group (Figure 6C). Moreover, at 4 months after irradiation, the size of macrophage that was stained with Mac-3 antibody was the larger than that of normal mice. These data suggested that macrophages that were recruited into the lung were in a state of activation and differentiation according to microenvironments that were changed after thoracic irradiation.

\section{Recruited macrophages after thoracic irradiation are alternatively activated macrophages, M2 macrophages}

Generally, activating macrophages have been described two subsets with distinct functions [17-19]: M1 macrophages and M2 macrophages. Zhang and colleagues have reported
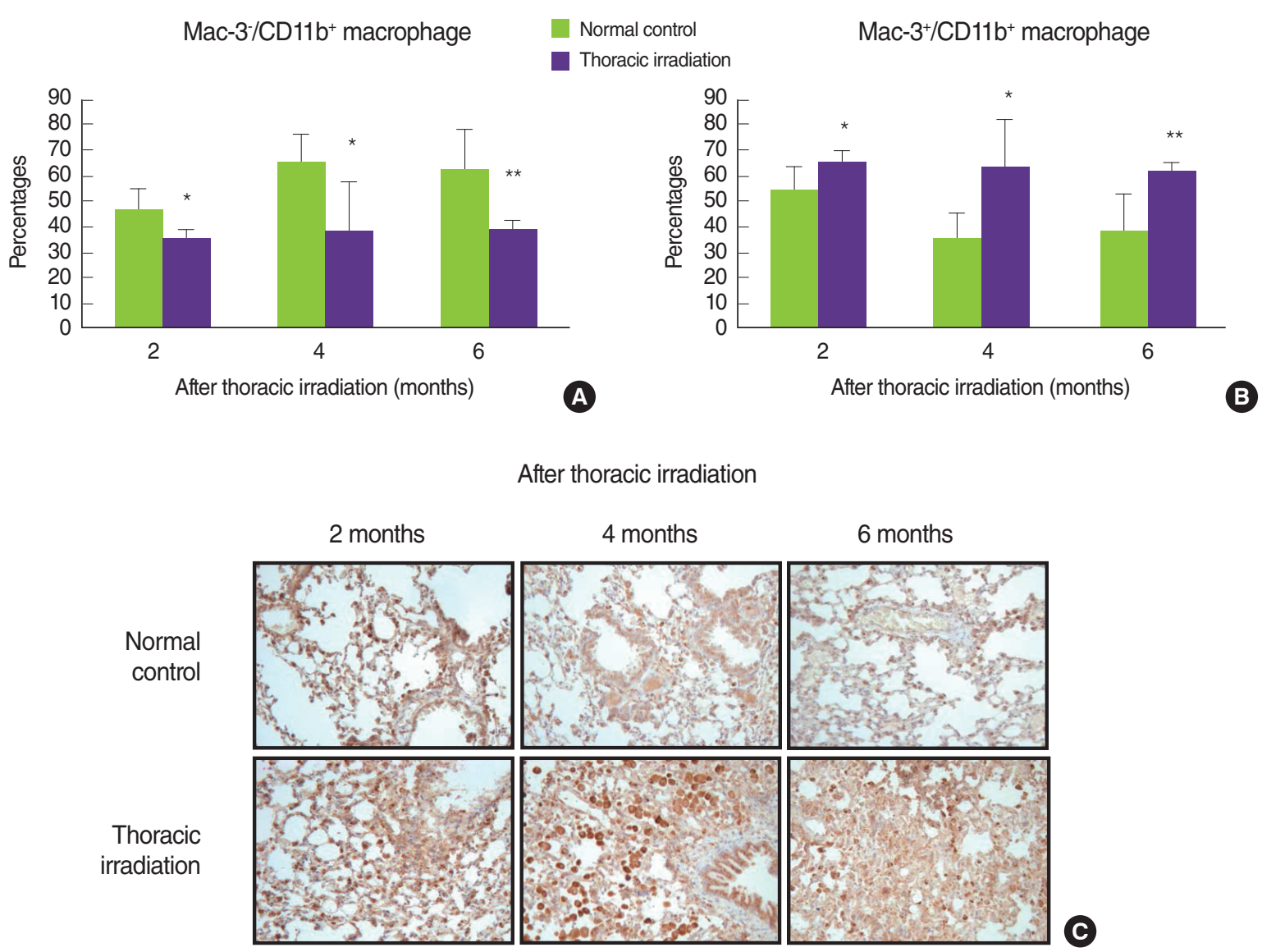

Fig. 6. Recruited macrophages after thoracic irradiation have Mac-3 antigen on their surface. At 2, 4, and 6 months after thoracic irradiation, total leukocytes were prepared from the lung and the lung tissues were fixed in $10 \%$ neutral-buffered formalin. (A, B) leukocytes in the lung were stained with anti-CD11b mAb and anti-Mac-3 mAb and then were analyzed by flow cytometer. There were six mice in each group. The bars indicate the means $\pm S D$. (C) Mac-3 positive macrophages in the lung tissues were determined by immunohistiochemical staining as described in the Materials and Methods section. Positive staining is depicted in brown ( $\times 400$ magnification). ${ }^{*} p<0.05$; ${ }^{* *} p<0.01$ compared to the age-matched control mice. 
that classical and alternative mechanisms of activating macrophages play a role during the pneumonic stage and the fibrotic phase, respectively [14]. Therefore, we examined what thoracic irradiation affects the polarization of activated macrophages that were recruited into the lung. As can be seen in Figure 7A, mRNA expressions of iNOS and CD32 in the lung at 4 months after thoracic irradiation very weak and no differences when compared with those in normal control mice but the expressions of iNOS protein were slightly decreased in thoracic irradiated mice. Conversely, thoracic irradiation increases the mRNA and protein expressions of Arg-1 and MMR (Figure 7A, 7B) relative to normal control mice. These data strongly suggest that macrophages infiltrated into the lung after thoracic irradiation were polarized into the alternatively activated macrophages, M2 macrophages. Interestingly, many studies have identified alternatively activated macrophages (AAMs; M2) as critical regulators of fibrosis $[14,17-19,31,32]$. After lung damage, an insufficient repair is associated with the persistence of M2 macrophages that continue to produce growth factors, which also stimulate fi- broblast activation and ECM secretion.

There are many studies in which IL-4 and IL-13 induced alternatively activated macrophages, which enhanced the expression of Arg-1 [19, 33, 34]. Therefore, we examined IL-4 and IL-13 levels in BAL fluid at 4 months following thoracic irradiation. As shown in Figure 7C, both IL-4 and IL-13 were markedly high levels in the BAL fluid of thoracic irradiated mice and there was significant difference between normal mice and thoracic irradiated mice $(121 \pm 40$ vs. $231 \pm 83 ; 115 \pm$ 42 vs. $217 \pm 92$, respectively). In contrast, expression levels of IFN- $\gamma$ were significantly decreased in thoracic irradiated mice $(p<0.01)$. Through our previous study, we thought that irradiation made the polarization of AAMs much easier, because irradiated mice were oriented into Th2-induced immunity as described above [35, 36]. Interestingly, IL-4 and IL-13 are powerful profibrotic cytokines through the direct stimulation of collagen synthesis in fibroblasts [31, 37]. For this reason, detailed mechanistic studies conducted with IL-4 and IL-13 inhibitors and IL-4 knock-out and IL13 knock-out mice have been happening to modulate pneumonitis and pulmonary
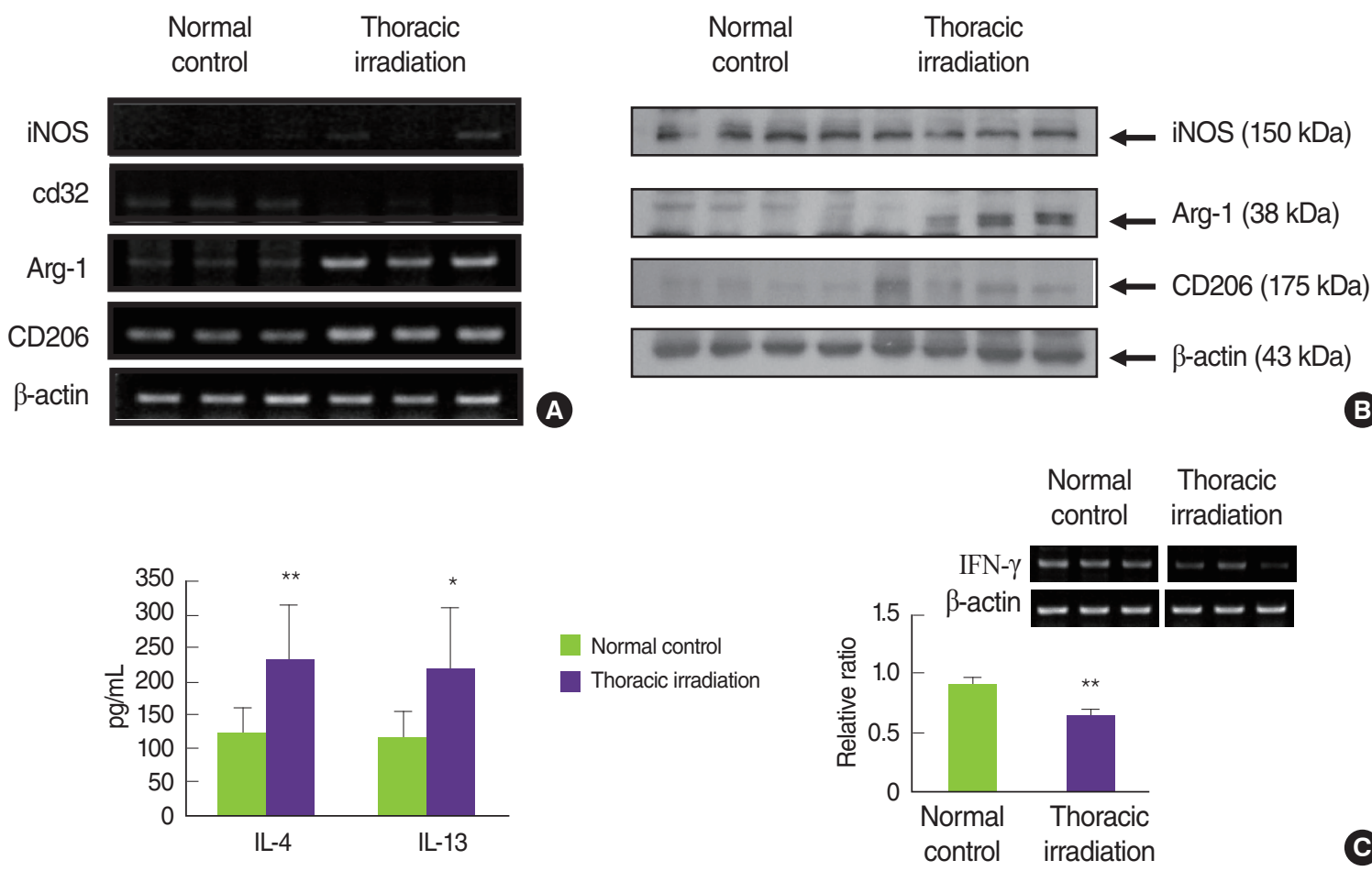

-

Fig. 7. Characteristic of macrophages infiltrated into the lung in thoracic irradiated mice. At 4 months following thoracic irradiation, BAL fluid and the lung tissues were collected. (A) The mRNA expression of iNOS, CD32, Arg-1, and CD206 was determined by RT-PCR methods and was quantitated by a Digital Gel Image system as described in the Materials and Methods section. There were three mice in each group. (B) The protein expressions of iNOS, Arg-1, and CD206 were determined by immunoblotting method. (C) IL-4 and IL-13 in BAL fluid were measured by ELISA and the mRNA expression of IFN- $\gamma$ in the lung was detected by RT-PCR. There were six mice in each group. The bars indicate the means $\pm \mathrm{SD}$. ${ }^{*} p<0.05 ;{ }^{* *} p<0.01$ compared to the age-matched control mice. 
fibrosis [38-41]. Recently, there are reports that loss of IL-4 and IL-13 is trying to control the stimulation of AAMs, and ultimately it overcomes pneumonitis and fibrosis [42-44]. Conversely, Th1 subsets of CD4+ T lymphocytes, defined by their production of IFN- $\gamma$, have antifibrotic activity [45]. Mechanistically, IFN- $\gamma$ inhibits the TGF- $\beta$-induced phosphorylation of the signal transducer Smad3 and subsequent activation of TGF- $\beta$-responsive genes and also directly inhibits fibroblast proliferation $[46,47]$. In our present results, the levels of IL-4 and IL-13 in the BAL fluid and the expression of Arg-1 and CD206 in the lung are elevated in thoracic irradiated mice, suggesting that macrophages infiltrated into the lung following thoracic irradiation are differentiated into the alternatively activated macrophages and these macrophages play an important role in radiation-induced fibrosis.

\section{Conclusion}

We reached the conclusion that alternatively activated macrophages must have played a very important role in the development of pneumonitis and fibrosis after thoracic radiation, although the detailed mechanisms by which they promote radiation-induced pneumonitis and fibrosis have not yet been resolved. Studies are ongoing to determine the mechanism by which alternatively activated macrophages and Th cells promote the development of pulmonary fibrosis following thoracic irradiation.

\section{Acknowledgements}

This work was conducted under the creative research project funded from Korea Atomic Energy Research Institute (KAERI), Korea (KAERI 527910-13).

\section{References}

1. Mehta V. Radiation pneumonitis and pulmonary fibrosis in nonsmall-cell lung cancer: pulmonary function, prediction, and prevention. Int. J. Radiat. Oncol. Biol. Phys. 2005;63:5-24.

2. Thannickal VJ, Toews GB, White ES, Lynch III JP, Martinez FJ. Mechanisms of pulmonary fibrosis. Ann. Rev. Med. 2004;55:395417.

3. Wynn TA. Integrating mechanisms of pulmonary fibrosis. J. Exp. Med. 2011;208:1339-1350.

4. Wilson MS, Wynn TA. Pulmonary fibrosis: pathogenesis, etiology and regulation. Mucosal Immunol. 2009;2:103-121.

5. Wynn TA. Cellular and molecular mechanisms of fibrosis. J.
Pathol. 2008;214:199-210.

6. Wynn TA, Ramalingam TR. Mechanisms of fibrosis: therapeutic translation for fibrotic disease. Nat. Med. 2012;18:1028-1040.

7. Duffield JS, et al. Selective depletion of macrophages reveals distinct, opposing roles during liver injury and repair. J. Clin. Invest. 2005;115:56-65.

8. Johnston CJ, Wright TW, Rubin P, Finkelstein JN. Alterations in the expression of chemokine mRNA levels in fibrosis-resistant and -sensitive mice after thoracic irradiation. Exp. Lung Res. 1998;24:321-337

9. Johnston CJ, Williams JP, Okunieff P, Finkelstein JN. Radiationinduced pulmonary fibrosis: examination of chemokines and chemokines receptor families. Radiat. Res. 2002;157:256-265.

10. Park HR, Jo SK, Yu DK, Jung UH. Fractionated irradiations lead to chronic allergic airway inflammation through increasing the influx of macrophages. Inflamm. Res. 2013;62:27-36.

11. Buttner $\mathrm{C}$, et al. Local production of interleukin-4 during radiation-induced pneumonitis and pulmonary fibrosis in rats: macrophages as a prominent source of interleukin-4. Am. J. Respir. Cell Mol. Biol. 1997;17:315-325.

12. Chiang CS, et al. Compartmental responses after thoracic irradiation of mice: strain differences. Int. J. Radiat. Oncol. Biol. Phys. 2005;62:862-871.

13. Yarnold J, Brontons MCV. Pathogenetic mechanisms in radiation fibrosis. Radiother. Oncol. 2010;97:149-161.

14. Zhang $\mathrm{H}$, et al. The development of classically and alternatively activated macrophages has different effects on the varied stages of radiation-induced pulmonary injury in mice. J. Radiat. Res. 2011;52:717-726.

15. Zaidi A, Jelveh S, Mahmood J, Hill RP. Effects of lipopolysaccharide on the response of $\mathrm{C} 57 \mathrm{BL} / 6 \mathrm{~J}$ mice to whole thorax irradiation. Radiother. Oncol. 2012;105:341-349.

16. Gordon S, Taylor PR. Monocyte and macrophage heterogeneity. Nat. Rev. Immunol. 2005;5:953-964.

17. Murray PJ, Wynn TA. Protective and pathogenic functions of macrophage subsets. Nat. Rev. Immunol. 2011;11:723-737.

18. Gordon S, Martinez FO. Alternative activation of macrophages: mechanism and functions. Immunity. 2010;32:593-604.

19. Mantovani A, Sica A, Sozzani S, Allavena P, Vecchi A, Locati M. The chemokines system in diverse forms of macrophage activation and polarization. Trends Immunol. 2004;12:677-686.

20. Nair MG, Cochrane DW, Allen JE. Marophages in chronic type 2 inflammation have a novel phenotype characterized by the abundant expression of Yml and Fizz1 that can be partly replicated in vitro. Immunol. letters. 2003;85:173-180.

21. Jackson IL, Vujaskovic Z, Down JD. Revisiting strain-related differences in radiation sensitivity of the mouse lung: recognizing and avoiding the confounding effects of pleural effusions. Radiat. Res. 2010;173:10-20.

22. Dileto CL, Travis EL. Fibroblast radiosensitivity in vitro and lung 
fibrosis in vivo: comparison between a fibrosis-prone and fibrosis-resistant mouse strain. Radiat. Res. 1996;146:61-67.

23. Franko AJ, Sharplin J, Chahary A, Barcellos-Hoff MH. Immunohistochemical localization of transforming growth factor $\beta$ and tumor necrosis factor $\alpha$ in the lungs of fibrosis-prone and "nonfibrosing" mice during the latent period and early phase after irradiation. Radiat. Res. 1997;147:245-256.

24. Johnston CJ, Piedboeuf B, Baggs R, Rubin P, Finkelstein JN. Differences in correlation of mRNA gene expression in mice sensitive and resistant to radiation-induced pulmonary fibrosis. Radiat. Res. 1995;142:197-203

25. Johnston CJ, Wiliams JP, Elder A, Hernady E, Finkelstein JN. Inflammatory cell recruitment following thoracic irradiation. Exp. Lung Res. 2004;30:369-382.

26. Rubin P, Finkelstein JN, Shapiro D. Molecular biology mechanisms in the radiation induction of pulmonary injury syndromes: interrelationship between the alveolar macrophage and the septal fibroblast. Int. J. Radiat. Oncol. Biol. Phy. 1992;24: 93-101.

27. Gabbiani G. The myofibroblasts in wound healing and fibrocontractive diseases. J. Pathol. 2003;200:500-503.

28. Dougherty GJ, McBride WH. Macrophage heterogeneity. J. Clin. Lab. Immunol. 1984;14:1-11.

29. Springer TA. Monoclonal antibody analysis of complex biological systems. Combination of cell hybridization and immunoadsorbents in a novel cascade procedure and its application to the macrophage cell surface. J. Biol. Chem. 1981;256:3833-3839.

30. Ho MK, Springer TA. Tissue distribution, structural characterization, and biosynthesis of Mac-3, a macrophage surface glycoprotein exhibiting molecular weight heterogeneity. J. Biol. Chem. 1983;258:636-642.

31. Sun L, et al. New concepts of IL-10-induced lung fibrosis: fibrocytes recruitment and M2 activation in a CCL2/CCR2 axis. Am. J. Physiol. Lung Cell Mol. Physiol. 2011;300:L341-L353.

32. Varin A, Goron S. Alternative activation of macrophages: immune function and cellular biology. Immunobiology. 2009;214: 630-641.

33. Mosser DM, Edwards JP. Exploring the full spectrum of macrophage activation. Nat. Rev. Immunol. 2008;8:958-969.

34. Anthony RM, et al. Memory T(H)2 cells induce alternatively activated macrophages to mediate protection against nematode parasites. Nat. Med. 2006;12:955-960.

35. Park HR, Jo SK, Paik SG. Factors effecting the Th2-like immune response after gamma-irradiation: low production of IL-12 het- erodimer in antigen-presenting cells and small expression of the IL-12 receptor in T cells. Int. J. Radiat. Biol. 2005;81:221-231.

36. Park HR, Jo SK, Eom HS. Chronic effects of single and fractionated $\gamma$-irradiation on an impairment of Thl-related immune response. Int. J. Radiat. Biol. 2011;87:534-543.

37. Song E, Ouyang N, Horbelt M, Antus B, Wang M, Exton MS. Influence of alternatively and classically activated macrophages on fibrogenic activities of human fibroblasts. Cell. Immunol. 2000;204:19-28.

38. Chiaramonte MG, Donaldson DD, Cheever AW, Wynn TA. An IL-13 inhibitor blocks the development of hepatic fibrosis during a T-helper type 2-dominated inflammatory response. J. Clin. Invest. 1999;104:777-785.

39. Huaux F, Liu T, McGarry B, Ullenbruch M, Phan SH. Dual roles of IL-4 in lung injury and fibrosis. J. Immunol. 2003;170:20832092.

40. Kolodsick JE, et al. Protection from fluorescein isothiocyanateinduced fibrosis in IL-13-deficient, but not IL-4-deficient, mice results from impaired collagen synthesis by fibroblasts. J. Immunol. 2004;172:4068-4076.

41. Yang G, et al. Anti-IL-13 monoclonal antibody inhibits airway hyperresponsiveness, inflammation and airway remodeling. Cytokine 2004; 28:224-232.

42. Lumsden RV, et al. Modulation of pulmonary fibrosis by IL13Ra2. Am. J. Physiol. Lung Cell Mol. Physiol. 2015;308:L710L718.

43. Su S, Zhao Q, et al. miR-142-5p and miR-130a-3p are regulated by IL-4 and IL-13 and control profibrogenic macrophage program. Nat. Commun. 2015;6:8523.

44. Groves AM, Johnston CJ, Misra RS, Williams JP, Finkelstein JN. Effects of IL-4 on pulmonary fibrosis and the accumulation and phenotype of macrophage subpopulations following thoracic irradiation. Int. J. Radiat. Biol. 2016;92:754-765.

45. Giri SN, Hyde DM, Marafino BJ Jr. Ameliorating effect of murine interferon- $\gamma$ on bleomycin-induced lung collagen fibrosis in mice. Biochem. Med. Meta. Biol. 1986;36:194-197.

46. Gurujeyalakshmi G, Giri SN. Molecular mechanisms of antifibrotic effect of interferon- $\gamma$ in bleomycin mouse model of lung fibrosis: downregulation of TGF- $\beta$ and procollagen I and III gene expression. Exp. Lung Res. 1995;21:791-808.

47. Ulloa L, Doody J, Massague J. Inhibition of transforming growth factor- $\beta /$ SMAD signaling by the interferon $-\gamma /$ STAT pathway. Nature. 1999;397:710-713. 